\title{
Downregulation of connective tissue growth factor reduces migration and invasiveness of osteosarcoma cells
}

\author{
YINJUN HUANG* , SHICHANG ZHAO*, CHANGQING ZHANG and XIAOLIN LI \\ Department of Orthopedic Surgery, Shanghai Sixth People's Hospital, Shanghai Jiao Tong University, \\ Shanghai 200233, P.R. China
}

Received November 10, 2014; Accepted September 18, 2015

DOI: $10.3892 / \mathrm{mmr} .2015 .4701$

\begin{abstract}
As one of the most serious types of primary bone tumor, osteosarcoma (OSA) features metastatic lesions, and resistance to chemotherapy is common. The underlying mechanisms of these characteristics may account for the failure of treatments and the poor prognosis of patients with OSA. It has been reported that inhibition of Cyr61 suppresses OSA cell proliferation as it represents a target of statins. In addition to cystein-rich protein 61 (Cyr61) and nephroblastoma overexpression, connective tissue growth factor (CTGF) is a member of the CCN family and may therefore exhibit effects on human OSA cells similar to those of Cyr61. In the current study, acridine orange/ethidium bromide staining were used to determine the rate of apoptosis. The present study demonstrated that small interfering RNA-mediated silencing of CTGF promoted cell death and suppressed OSA cell migration and invasion, as indicated by wound healing and Transwell assays, while lentivirus-mediated overexpression of CTGF reversed these effects. Furthermore, a colorimetric caspase assay demonstrated that CTGF knockdown enhanced the efficacy of chemotherapeutic drugs. The results of the present study
\end{abstract}

Correspondence to: Professor Xiaolin Li, Department of Orthopedic Surgery, Shanghai Sixth People's Hospital, Shanghai Jiao Tong University, 600 Yishan Road, Shanghai 200233, P.R. China E-mail: lixiaolin@sjtu.edu.cn

*Contributed equally

Abbreviations: Cyr61, cysteine-rich angiogenic inducer 61; CTGF, connective tissue growth factor; NOV, nephroblastoma-overexpressed gene protein homolog; OSA, osteosarcoma; MDM2, mouse double minute 2 homolog; Rb, retinoblastoma gene; HES1, hes family BHLH transcription factor 1; MMLV, moloney murine leukemia virus reverse transcriptase; dNTP, deoxynucleoside triphosphate; qPCR, quantitative polymerase chain reaction; GTPase, hydrolase enzymes of guanosine triphosphate; FTase, farnesyltransferase; FPP, farnesyl pyrophosphate; HMGCoA, 3-hydroxy-3-methyl glutaryl coenzyme A; GGPP, geranylgeranyl pyrophosphate

Key words: connective tissue growth factor, statin, invasion, migration, chemotherapy resistance provided a novel molecular target which may be utilized for the treatment of metastatic OSA.

\section{Introduction}

Osteosarcoma (OSA) is globally one of the most common types of primary bone tumor and is predominantly observed in children and adolescents (<20 years old) (1). Patients with localized disease have a five-year recurrence-free survival rate of $80 \%$; however, the prognosis of OSA is poor in metastatic osteosarcoma. In spite of OSA occurring in any type of bone in the body, the metaphyseal (actively growing) regions of the distal femur, proximal tibia and proximal humerus are the most frequent origins of the primary tumor and the sites with the highest probability of metastasis are the lungs and distant bones (2).

It has been reported that several genes are able to regulate cell proliferation and differentiation; these genes carry numerous mutations associated with significant neoplasmic abnormalities in OSA (3-9). Of note, mutations in tumor suppressor genes, including p53, MDM2 and riboblastoma protein have been reported to have major roles in the tumorigenesis of OSA $(3,4)$. OSA is also associated with the aberrant expression of certain transcription factors expressed in bones, including c-fos, whose overexpression has been shown to result in OSA in the bones of mice (5), as well as osteoblast differentiation factor osterix (6,7). In OSA cell lines, Runx2 was found to be downregulated or dysfunctional (8), and in high-grade pediatric OSA, genomic aberrations in the Twist have been reported (9).

Resistance to conventional chemotherapy is one of the characteristics of metastatic OSA and represents a considerable obstacle for its clinical treatment (10). However, only a small number of genes, including HES1 (11-13) and Ezrin (10) have been implicated in the progression and metastasis of OSA.

It has been reported that statins exert anti-tumoral effects on OSA cells (13-15). Cystein-rich protein 61 (Cyr61), a member of the Cyr61/connective tissue growth factor (CTGF)/nephroblastoma overexpressed (NOV) (CCN) family of secreted proteins, was among the factors downregulated by statins. This CCN protein family comprises Cyr61, CTGF, NOV and Wnt-induced secreted proteins (WISP)1, -2 and -3 (16). As a member of the CCN family, CTGF was 
hypothesized have effects on osteocarcinoma similar to those of statins. The present study therefore assessed the effects of CTGF knockdown or lentivirus-mediated overexpression of CTGF as well as statin treatment on the biological properties of OSA cells.

\section{Materials and methods}

Cell lines and culture. The SaOS2, U2OS, MG63, OHS4 and CAL72 cell lines (American Type Culture Collection, Manassas, VA, USA) were cultured in Dulbecco's modified Eagle's medium (Invitrogen; Thermo Fisher Scientific, Waltham, MA, USA) supplemented with $10 \%$ fetal calf serum (FCS; Thermo Fisher Scientific) at $37^{\circ} \mathrm{C}$ in a humidified atmosphere containing $5 \% \mathrm{CO}_{2}$ in air.

RNA extraction and reverse-transcription quantitative polymerase chain reaction ( $R T-q P C R)$. TRIzol reagent (Invitrogen; Thermo Fisher Scientific) was used to isolate RNA according to the manufacturer's instructions, which was stored at $-20^{\circ} \mathrm{C}$. cDNA was synthesized using $3 \mu \mathrm{g}$ RNA, which was denaturated and reverse-transcribed by using $300 \mathrm{U}$ Moloney murine leukemia virus reverse transcriptase, $15 \mathrm{mg}$ oligo dT primers and $1 \mathrm{mM}$ deoxynucleoside triphosphate (dNTP) (Promega, Madison, WI, USA) in a total volume of $30 \mu \mathrm{l}$. SYBR Green Master Mix kit (ABGen, Courtaboeuf, France) was used for qPCR. A total of $0.5 \mathrm{mM}$ of each primer (Invitrogen; Thermo Fisher Scientific) was used with sequences as follows: Human CTGF, forward 5'-CAGGCTAGAGAAGCAGAG CC-3' and reverse 5'-GTAATGGCAGGCACAGGTCT-3'; $\beta$-actin, forward 5'-CTCCATCCTGGCCTCGCTGT-3' and reverse 5'-GCTGTCACCTTCACCGTTCC-3'. Thermocycling was conducted using the ABI 7500 (Applied Biosystems; Thermo Fisher Scientific) and the cycling conditions were as follows: Initial denaturation at $95^{\circ} \mathrm{C}$ for $15 \mathrm{~min}$, followed by 40 cycles of $20 \mathrm{sec}$ at $95^{\circ} \mathrm{C}, 15 \mathrm{sec}$ at $58^{\circ} \mathrm{C}$ and $15 \mathrm{sec}$ at $72^{\circ} \mathrm{C}$, and final extension at $72^{\circ} \mathrm{C}$ for $7 \mathrm{~min}$. The $2^{-\Delta \Delta \mathrm{Ct}}$ method was used to determine the relative quantities of RNA.

Plasmid transduction. In order to investigate the role of CTGF in OSA, cell lines were transduced with lentiviral vectors (LV) encodingeitherthefull-length sequence(LV-CTGF) oraspecific short hairpin (sh)RNA (sh-CTGF). The full-length CTGF ORF (1047 base pairs; GenBank accession number, CR541759.1) was amplified from the pFLAG-CMV2-CTGF plasmid (Invitrogen; Thermo Fisher Scientific). The primer sequences were as follows: Forward, 5'-TACTGGCGGCGGTATACCCG-3' and reverse, 5'-TGCCATGTCTCCGTACAT-3'. The PCR product was inserted into the expression vector pcDNA3.1/myc-His(-)B-3X FLAG-IRES-hrGFP, derived from pcDNATM3.1/myc-His(-)B (Invitrogen; Thermo Fisher Scientific). Cell transduction was performed using Lipofectamine 2000 (Invitrogen; Thermo Fisher Scientific) according to the manufacturer's instructions.

Proliferation assay. A bromodeoxyuridine (BrdU) incorporation assay was used to quantify cell replication. A previously described procedure was used in the present study (17). In brief, cells were cultured for $24 \mathrm{~h}$ in the presence of increasing concentrations of bisphosphonates $\left(10^{-9}-10^{-4} \mathrm{M}\right)$ and labeled with BrdU for the last $6 \mathrm{~h}$ (kit purchased from GE Healthcare Life Sciences, Roosendaal, The Netherlands).

Detection of apoptosis and necrosis. Double staining with ethidium bromide and acridine orange was performed to visualize and quantify the number of viable cells (green nuclei), apoptotic cells (nuclei condensed and colored orange), and necrotic cells (red nuclei). In briefly, $2 \mu 1$ dye mixture (100 $\mu \mathrm{g} / \mathrm{ml}$ acridine orange and $100 \mu \mathrm{g} / \mathrm{ml}$ ethidium bromide) was added to $20 \mu \mathrm{l}$ cell suspension and immediately examined with the 40X oil immersion objective using a Leitz DMRB fluorescence microscope (green/red filter; $100 \mathrm{~W}$ lamp; Leica Microsystems GmbH, Wetzlar, Germany) equipped with a photometrics CCD camera and the Logikon image analysis system (Numeris Benelux SA, Ath, Belgium). Several fields, randomly chosen, were digitized and 600-800 nuclei for each sample were counted and scored. Results were expressed as the relative percentages of viable, apoptotic and necrotic cells to the total number of cells scored.

Caspase activity. Effector caspase activity was performed as previously described $(14,15)$. In brief, cells were treated with $10 \mathrm{mM}$ atorvastatin (Adooq BioScience LLC, Irvine, CA, USA) or the solvent for $24 \mathrm{~h}$ then the caspase activity was determined. Cellular extracts $(50 \mu \mathrm{g})$ were incubated with $0.2 \mathrm{mM}$ acetyl-Asp-Glu-Val-Asp-p-nitroanilide (caspases-3, -6 and -7; Enzo Life Sciences, Inc., Farmingdale, NY, USA), Ac-LEHD-pNA (caspase-9; Enzo Life Sciences, Inc.) or Ac-IETD-pNA (caspase-8; Enzo Life Sciences, Inc.) as the substrates for the previously reported times $(14,15)$ at $37^{\circ} \mathrm{C}$ in the presence or the absence of the specific caspase inhibitors Ac-DEVD-CHO, Ac-LEHD-CHO and Ac-IETD-CHO $(10 \mu \mathrm{M})$. The specific activity (nmol of $\mathrm{pNA} / \mathrm{min} / \mathrm{mg}$ protein) was expressed as treated over control ratios.

Migration and invasion assays. A wound-healing assay was performed following the manufacturer's instructions (ibidi $\mathrm{GmbH}$, Martinsried, Germany). A Transwell migration and invasion assay as performed as described previously (14). In brief, the cells $(50,000$ cells/insert) were incubated $2 \mathrm{~h}$ with or without statin and/or z-VAD-fmk prior to seeding into the inserts and incubation for a further $22 \mathrm{~h}$. The cells that did not migrate through the filter were removed from the upper surface of the membrane using cotton-tipped swabs. The cells migrated to the underside were fixed in $3.7 \%$ paraformaldehyde in phosphate-buffered saline (PBS) at $4^{\circ} \mathrm{C}$ and stained with crystal violet (Amresco, Solon, OH, USA). The membranes were then cut from the insert and observed under a microscope (Axioplan 2 Imaging Mot Microscope System; Zeiss, Oberkochen, Germany). Five fields were randomly selected and counted and each assay was performed in duplicate.

Western blot analysis. A protocol of a previous study was used for the preparation of cell lysates (14) In brief, the proteins (30 $\mu \mathrm{g}$ ) were resolved using 12\% sodium dodecyl sulfate polyacrylamide gel electrophoresis (SDS-PAGE; Protogel, Atlanta, GA, USA) and transferred onto polyvinylidene difluoride nitrocellulose membranes (EMD Millipore, Billerica, MA, USA). The filters were incubated at room temperature for $2 \mathrm{~h}$ in $50 \mathrm{~mm}$ Tris- $\mathrm{HCl}$ (pH 7.4), $150 \mathrm{~mm} \mathrm{NaCl}, 0.1 \%$ (v/v) 

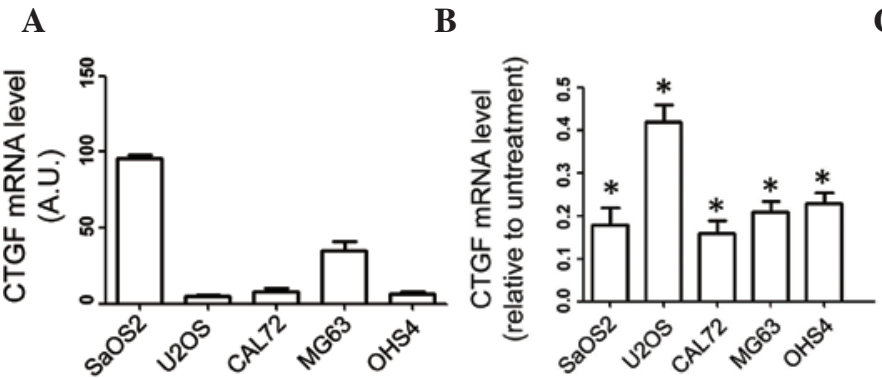

C

Figure 1. CTGF expression levels in osteosarcoma cell lines are reduced by atorvastatin. (A) RT-qPCR was performed to evaluate CTGF mRNA expression in several human OSA cell lines. Expression was normalized to $\beta$-actin. (B) Human OSA cell lines were treated with $10 \mathrm{mM}$ atorvastatin for $24 \mathrm{~h}$ and RT-qPCR was used to evaluate mRNA levels of CTGF. Relative expression levels compared with those in untreated cells are shown. Values are expressed as the mean \pm standard deviation $(n=3)$. " $P<0.05$ vs. A. (C) Immunoblot analysis was used to assess protein levels of CTGF in human OSA cell lines treated with $10 \mathrm{mM}$ atorvastatin for $24 \mathrm{~h}$. Ratios of protein expression compared with those of untreated cells are presented, as an average of three blots. Statin, atorvastatin; CTGF, connective tissue growth factor; A.U., arbitrary units; RT-qPCR reverse-transcription quantitative polymerase chain reaction.

Tween 20, 0.5\% (w/v) bovine serum albumin (TBST/BSA) and then overnight at $4^{\circ} \mathrm{C}$ on a shaker with the rabbit monoclonal anti-GAPDH (ab181602) and anti-CTGF (ab6992) antibodies (1:1,000 in TBST/BSA; Abcam, Cambridge, UK). The membranes were washed twice with TBST and incubated for $2 \mathrm{~h}$ with the horseradish peroxidase-conjugated secondary antibody (1:20,000 in TBST/BSA). Following the final washes, the signals were visualized with Enhanced Chemiluminescence Western Blotting Detection Reagent (GE Healthcare Life Sciences) and autoradiographic film (X-Omat AR; Kodak, Rochester, NY, USA). Densitometric analysis using ImageQuant software was performed following digital scanning (Odyssey ${ }^{\circledast}$ Fc; Agfa-Gevaert, Mortsel, Belgium).

Immunoblot analysis. A protocol of a previous study was used for the preparation of cell lysates $(14,15)$. Incubation with rabbit monoclonal anti-GAPDH (ab181602; 1:200) and rabbit polyclonal anti-CTGF (ab6992; 1:200) antibodies was conducted at $4^{\circ} \mathrm{C}$ overnight. Cell extracts were collected in $2 \mathrm{X}$ loading lysis buffer $[50 \mathrm{mM}$ Tris- $\mathrm{HCl}(\mathrm{pH} 6.8), 2 \%$ SDS, $10 \%$ 2-mercaptoethanol, $10 \%$ glycerol and protease inhibitor cocktail; Sigma-Aldrich, St. Louis, MO, USA]. The total cellular proteins were separated using $8 \%$ SDS-PAGE and trans- ferred to Hybond-C nitrocellulose membranes (GE Healthcare Life Sciences, Chalfont, UK). Subsequent to blocking with PBS containing 5\% BSA or nonfat milk, the membranes were incubated with the primary antibodies, followed by incubation with IRDye $800 \mathrm{CW}$ or $680 \mathrm{RD}$ secondary antibodies (1:10,000; LI-COR Biosciences, Lincoln, NE, USA). The protein bands were detected using the Odyssey Infrared Imaging System (Li-COR Biosciences).

Statistical analysis. Values are expressed as the mean \pm standard deviation. Two-factor analysis of variance was used to compare values between groups, using SPSS software, version 19.0 (IBM SPSS, Armonk, NY, USA). P $<0.05$ was considered to indicate a statistically significant difference between values.

\section{Results}

CTGF expression is reduced by atorvastatin (statin) in OSA cells. RT-qPCR analysis of CTGF was performed in the
SaOS2, U2OS, CAL72, MG63 and OHS4 human OSA cell lines, revealing that CTGF mRNA was expressed in all cell lines, particularly in SaOS2 cells (Fig. 1A). Furthermore, the effect of statin treatment on the expression of CTGF was assessed in the OSA cell lines. CTGF mRNA expression in the panel of OSA cell lines was markedly decreased following treatment with statin $(10 \mathrm{mM})(\mathrm{P}<0.05$ vs. untreated) (Fig. 1B). In addition, the effect of statin $(10 \mathrm{mM})$ on the protein levels of CTGF in the panel of cell lines was assessed by immunoblot analysis, revealing that the protein levels of CTGF were decreased following statin (Fig. 1C). Collectively, these results indicated that statin treatment led to the downregulation of CTGF in human OSA cells. As the SaOS2 and U2OS cell lines expressed the highest and lowest levels of CTGF, respectively, they were selected to be used in the subsequent experiments.

CTGF expression does not affect OSA cell proliferation. A BrdU incorporation assay were used to determine the proliferative rates of transduced and parental cells, revealing that these were not affected by plasmid transduction (Fig. 2A). The results therefore indicated that CTGF had no significant effects on OSA-cell proliferation in human cell lines.

\section{Evasion of apoptosis by OSA cells is dependent on CTGF} expression. The present study investigated the effects of CTGF on OSA cell death. As shown in Fig. 2B, apoptotic and necrotic indices of sh-CTGF-transduced cells were higher than those of parental cells. By contrast, LV-CTGF-transduced cells displayed lower apoptotic and necrotic indices compared with those of parental cells. Furthermore, it was revealed that sh-CTGF-transduced cells exhibited increased caspase activity and an elevated $\mathrm{Bax} / \mathrm{Bcl} 2$ ratio compared with those of parental cells. By contrast, caspase activity and the $\mathrm{Bax} / \mathrm{Bcl} 2$ ratio were reduced in CTGF-overexpressing OSA cells compared with those in parental cells (Fig. 2C and D). These results indicated that CTGF expression was associated with the evasion of apoptosis by OSA cells.

The dose-dependent cytotoxic effects of doxorubicin, cisplatin and methotrexate on OSA cell viability are utilized for the chemotherapeutic treatment of OSA (14). The present study revealed that CTGF silencing significantly enhanced the caspase activity in $\mathrm{SaOS} 2$ cells following treatment with doxorubicin, cisplatin or methotrexate, whereas LV-CTGF 


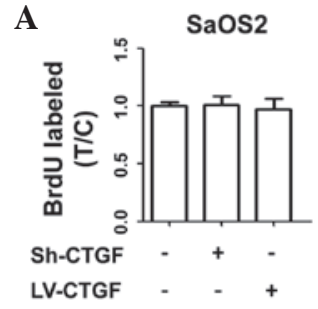

B

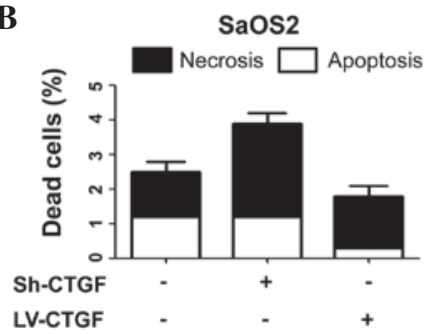

C
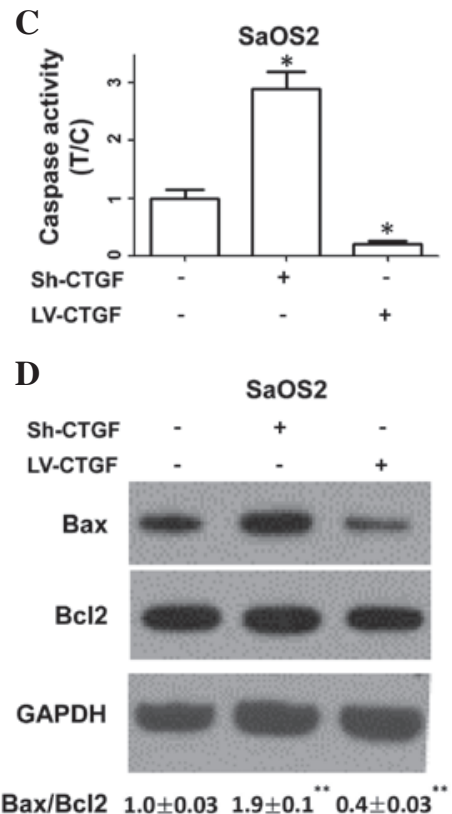
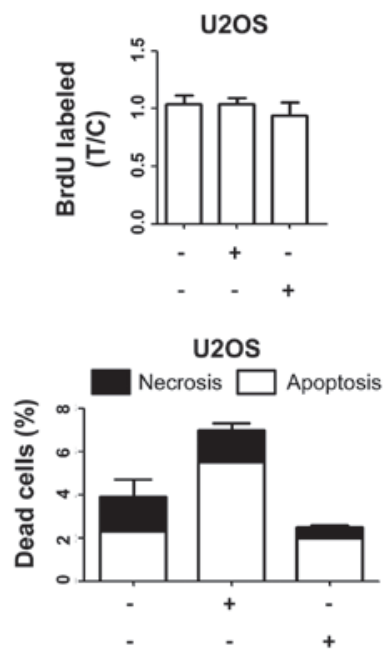

U2OS

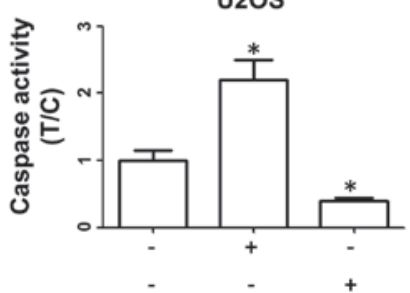

U2OS

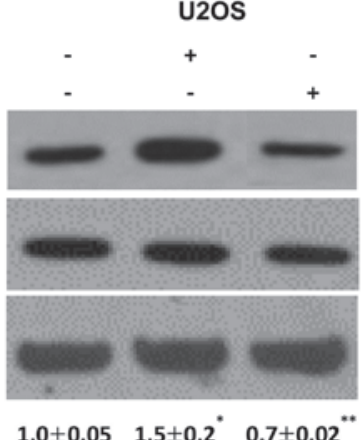

Figure 2. Osteosarcoma cell viability is modulated by CTGF expression. (A) Following $48 \mathrm{~h}$ of BrdU incorporation, cell proliferation was evaluated. (B) Acridine orange/ethidium bromide staining were performed and staining intensity was scored to determine the numbers of viable, apoptotic and necrotic cells. Necrotic and apoptotic cells are expressed as a percentage of the total cell population. (C) A colorimetric assay was used to evaluate caspase activity. (D) Immunoblot analysis was used to assess levels of apoptosis-associated proteins Bcl-2 and Bax in cell lysates. Blots were quantified to determine the $\mathrm{Bax} / \mathrm{Bcl} 2$ ratio. ${ }^{*} \mathrm{P}<0.05$ versus parental cells. Values are expressed as the mean \pm standard deviation $(\mathrm{n}=3)$. Bcl2, B-cell lymphoma 2; Bax, Bcl-2-associated X protein; LV, lentivirus; Sh, short-hairpin RNA; BrdU, bromodeoxyuridine; CTGF, connective tissue growth factor; $\mathrm{T} / \mathrm{C}$, treatment/control.

slighly decreased caspase levels compared with those in native $\mathrm{SaOS} 2$ cells treated with the chemotherapeutics (Fig. 3A-C). It is therefore concluded that silencing of CTGF enhanced the efficacy of chemotherapeutic drugs against OSA.

Cellmigration andinvasionare dependenton CTGF expression in vitro. The present study further investigated the invasiveness and migratory potential of transduced OSA cell lines, which represent the main characteristics of OSA progression and the development of metastasis. The results showed that CTGF silencing inhibited wound healing in sh-CTGF-transduced

A

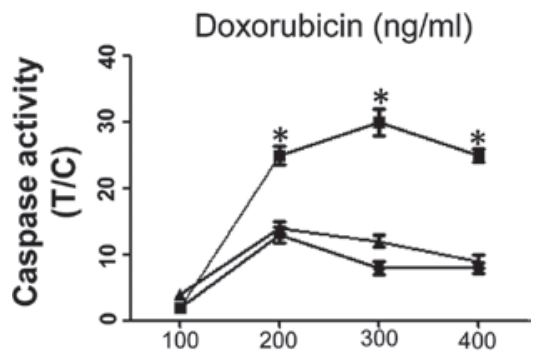

B

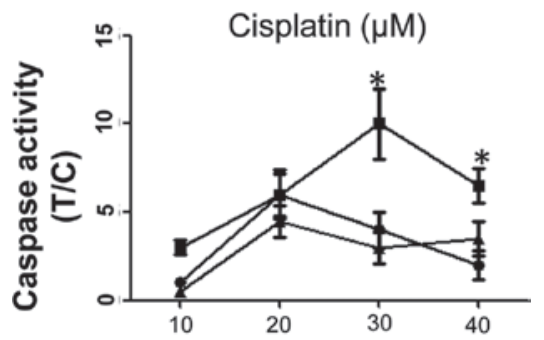

C

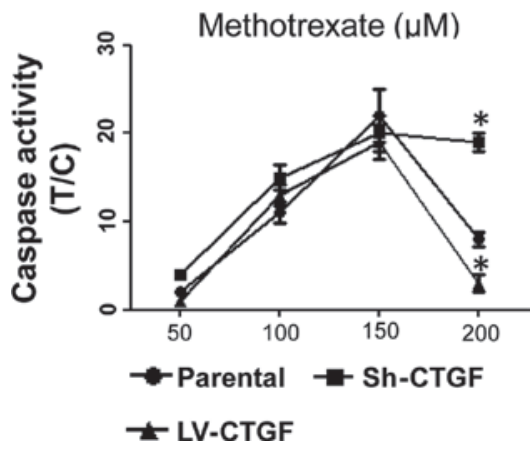

Figure 3. Efficacy of chemotherapeutics in OSA cells is enhanced by CTGF knockdown. Transduced SaOS2 cells were incubated with various doses of (A) doxorubicin, (B) cisplatin or (C) methotrexate. A colorimetric assay was used to evaluate caspase activity. " $\mathrm{P}<0.05$ vs control. Values are expressed as the mean \pm standard deviation $(\mathrm{n}=3)$. LV, lentivirus; Sh, short-hairpin RNA; CTGF, connective tissue growth factor; T/C, treatment/control.

cells compared with that in parental cells, while CTGF overexpression enhanced wound healing (Fig. 4A). In addition, CTGF overexpression enhanced the migratory potential in a Transwell assay (Fig. 4B). The observed inhibition of the migratory potential by statin was not able to be rescued by overexpression of CTGF (Fig. 4C). Furthermore, a Transwell assay using Matrigel-coated inserts revealed that silencing of CTGF inhibited the invasive capacity of OSA cells, while cell invasiveness was promoted by CTGF overexpression (Fig. 4D). All of these results implied that CTGF had positive effects on cell migration and invasiveness in vitro, whereas invasion and migration were reduced in CTGF-silenced OSA cells. It can be concluded that CTGF expression is associated with the aggressiveness and metastatic potential of OSA cells.

\section{Discussion}

Conserved cysteine residues covalently bound to isoprenoids can be post-translationlly modified by prenylation, which is essential for the pro-tumorigenic activity of certain guanosine triphosphatases, including Ras and Rho-like proteins $(18,19)$. Synthetic bisphosphonates with inhibitory activities on geranylgeranyltransferase type and farnesyltransferase can be utilized 
A

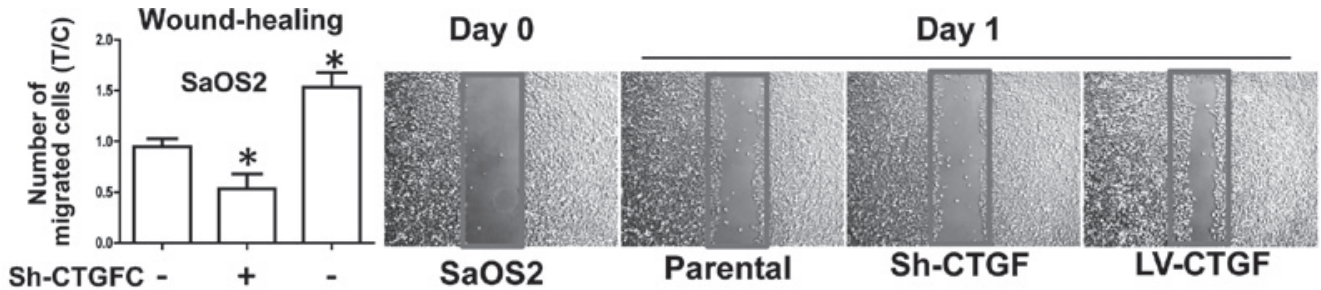

LV-CTGFC - $\quad$ - $\quad+$

B

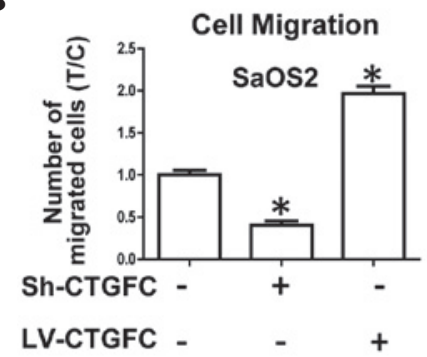

C
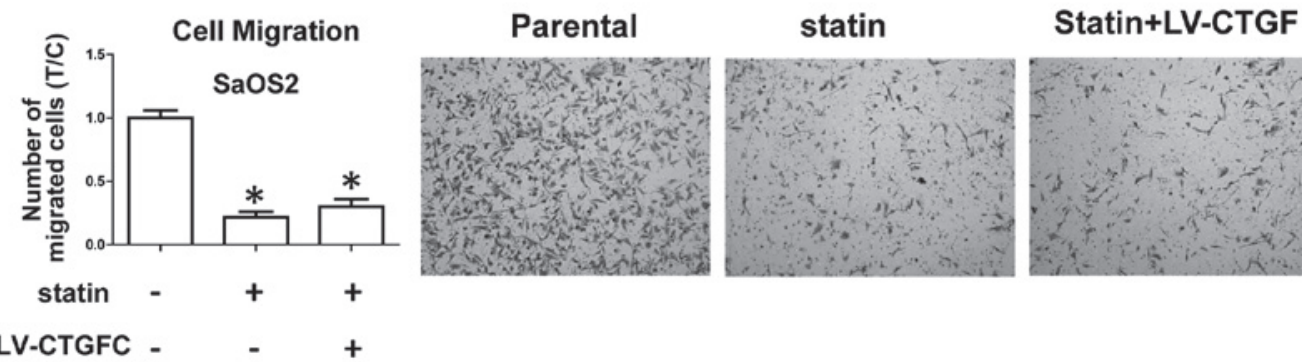

D
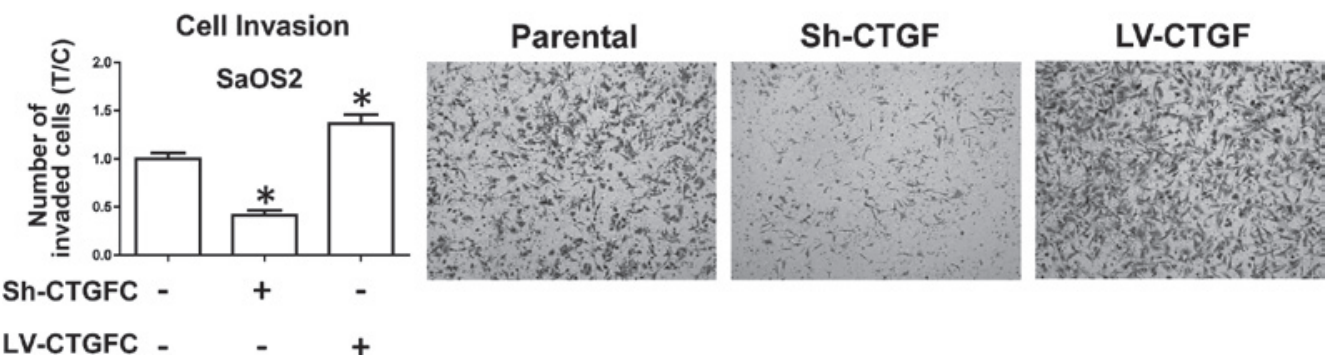

Figure 4. OSA cell migration and invasion are affected by CTGF expression. SaOS2 cells were subjected to CTGF knockdown, plasmid-mediated overexpression of GTGF and/or statin treatment. (A) A wound healing assay was used to assess the migratory potential of SaOS2 cells was assessed at one day one after wounding. Phase-contrast microscopy images were captured on days 0 and 1 after wounding. (B and C) A 24-h Transwell assay was used to assess the migratory capacity of SaOS2 cells. (D) The invasive capacity of SaOS2 cells was assessed using a 24-h Transwell assay using Matrigel-coated inserts. Values are expressed as the mean \pm standard deviation determined from eight fields of view. Magnification, $\mathrm{x} 40$; stained with crystal violet. "P<0.05 vs. parental cells. Statin, atorvastatin; LV, lentivirus; Sh, short-hairpin RNA; CTGF, connective tissue growth factor; T/C, treatment/control.

as anti-cancer drugs which partly block prenylation through inhibition of farnesyl pyrophosphate (FPP) synthase activity; this approach is a novel therapeutic strategy for several cancer types, including OSA and bone metastases (20-25). Statins act as hypocholesterolemic agents with inhibitory effects on the activity of 3-hydroxy-3-methylglutaryl-coenzyme A reductase (26) and represent another class of drug which acts through depleting downstream isoprenoid residues, including such as geranylgeranylpyrophosphate or FPP. Previous studies on OSA reported that statins not only induced apoptosis but also reduced cell migration and invasion, and potentiated the effects of chemotherapeutic agents (13-15). However, the anti-cancer efficacy of statins in vivo remains to be clarified. Previous clinical studies indicated that statins, apart from exhibiting anti-cancer effects, may also be associated with an increased risk for the development of cancer de novo (27-29).
These conflicting results indicate that the understanding of the mechanisms of action of statins is required to be expanded and refined, and that novel targets for cancer therapy require to be discovered.

Previous studies reported that Cyr61, which encodes a secreted protein known to modulate tumor development and progression, was downregulated by statins (30-32) and that CTGF is also among the molecular targets of statins $(33,34)$. CTGF is a matricellular protein of the CCN family of extracellular matrix-associated heparin-binding proteins, which comprises Cyr61, CTGF, NOV and WISP1-3 (35-37). CTGF has important roles in numerous biological processes, including cell adhesion, migration, proliferation, angiogenesis, skeletal development and tissue wound repair, and is critically involved in fibrotic disease and several types of cancer $(33,34,38)$. Members of the $\mathrm{CCN}$ protein family have similar domains, 
indicating that CTGF may have the similar roles in OSA cells to those of Cyr61.

The present study enhanced or silenced the expression of CTGF in human OSA cells to determine the role of CTGF in OSA development and progression. A BrdU incorporation assay did not reveal any significant effects of CTGF on the proliferation of human OSA cell lines. By contrast, CTGF silencing slightly increased OSA cell death and enhanced the anti-neoplasic and pro-apoptotic effects of the chemotherapeutics doxorubicin, cisplatin and methotrexate, which may represent a novel strategy to enhance the efficacy of OSA treatments. A positive combinatory effect of statins with chemotherapeutic drugs in OSA or other cancer types has been indicated by previous studies $(13,39-42)$. The present study focused on CTGF expression in OSA cells, independent of the presence of statins. As silencing of CTGF enhanced the anti-tumoral effects chemotherapeutic drugs, it was indicated that CTGF knockdown may reduce the resistance of OSA cells to chemotherapy.

OSA bears the characteristics of rapid and frequent development of metastatic lesions. In vitro experiments performed in the present study demonstrated that the migratory and invasive capacities of human OSA cells were reduced by CTGF silencing, whereas CTGF overexpression led to an increase in cell migration and invasion. By contrast, previous studies reported that silencing or inhibition of CTGF reduced the motility and invasiveness of breast and prostate cancer cells $(43,44)$. Due to this discrepancy, the roles of CCN family proteins, particularly CTGF, in OSA require further study. In OSA cell lines, Nov was reported to be expressed at variable levels (45) and may be associated with poor prognosis and an increased risk of developing metastases (46). The predictive value of CTGF expression levels with regard to the outcome and progression of human OSA requires to be investigated in future studies analyzing CTGF expression in primary and metastatic tumors.

In conclusion, the results of the present study revealed that OSA cell invasion and migration was regulated by CTGF in vitro. CTGF was indicated to have a critical role in the genesis and progression of human OSA, and to be involved in the evasion of apoptosis, aggressiveness and metastasis formation of OSA. Targeting of CTGF may be a strategy to enhance the efficacy of chemotherapeutics in the treatment of OSA as well as to reduce the aggressiveness of OSA cells.

\section{References}

1. Fromigue O, Hamidouche Z, Vaudin P, Lecanda F, Patino A, Barbry P, Mari B and Marie PJ: CYR61 downregulation reduces osteosarcoma cell invasion, migration, and metastasis. J Bone Miner Res 26: 1533-1542, 2011.

2. Zhang Y, Zhang L, Zhang G, Li S, Duan J, Cheng J, Ding G, Zhou C, Zhang J, Luo P, et al: Osteosarcoma metastasis: Prospective role of ezrin. Tumour Biol 35: 5055-5059, 2014.

3. Pompetti F, Rizzo P, Simon RM, Freidlin B, Mew DJ, Pass HI, Picci P, Levine AS and Carbone M: Oncogene alterations in primary, recurrent and metastatic human bone tumors. J Cell Biochem 63: 37-50, 1996.

4. Ladanyi M, Cha C, Lewis R, Jhanwar SC, Huvos AG and Healey JH: MDM2 gene amplification in metastatic osteosarcoma. Cancer Res 53: 16-18, 1993.

5. Wang ZQ, Liang J, Schellander K, Wagner EF and Grigoriadis AE: c-fos-induced osteosarcoma formation in transgenic mice: Cooperativity with c-jun and the role of endogenous c-fos. Cancer Res 55: 6244-6251, 1995.
6. Cao Y, Jia SF, Chakravarty G, de Crombrugghe B and Kleinerman ES: The osterix transcription factor down-regulates interleukin-1 alpha expression in mouse osteosarcoma cells. Mol Cancer Res 6: 119-126, 2008.

7. Cao Y, Zhou Z, de Crombrugghe B, Nakashima K, Guan H, Duan X, Jia SF and Kleinerman ES: Osterix, a transcription factor for osteoblast differentiation, mediates antitumor activity in murine osteosarcoma. Cancer Res 65: 1124-1128, 2005.

8. Thomas DM, Johnson SA, Sims NA, Trivett MK, Slavin JL, Rubin BP, Waring P, McArthur GA, Walkley CR, Holloway AJ, et al: Terminal osteoblast differentiation, mediated by runx 2 and p27KIP1, is disrupted in osteosarcoma. J Cell Biol 167: 925-934, 2004.

9. Entz-Werlé N, Stoetzel C, Berard-Marec P, Kalifa C, Brugiere L, Pacquement H, Schmitt C, Tabone MD, Gentet JC, Quillet R, et al: Frequent genomic abnormalities at TWIST in human pediatric osteosarcomas. Int J Cancer 117: 349-355, 2005.

10. O'Farrill JS and Gordon N: Autophagy in osteosarcoma. Adv Exp Med Biol 804: 147-160, 2014.

11. Zhang P, Yang Y, Zweidler-McKay PA and Hughes DP: Critical role of notch signaling in osteosarcoma invasion and metastasis. Clin Cancer Res 14: 2962-2969, 2008.

12. Khanna C, Wan X, Bose S, Cassaday R, Olomu O, Mendoza A, Yeung C, Gorlick R, Hewitt SM and Helman LJ: The membrane-cytoskeleton linker ezrin is necessary for osteosarcoma metastasis. Nat Med 10: 182-186, 2004.

13. Fromigué O, Hamidouche $\mathrm{Z}$ and Marie PJ: Statin-induced inhibition of 3-hydroxy-3-methyl glutaryl coenzyme a reductase sensitizes human osteosarcoma cells to anticancer drugs. J Pharmacol Exp Ther 325: 595-600, 2008.

14. Fromigué $\mathrm{O}$, Hamidouche $\mathrm{Z}$ and Marie PJ: Blockade of the RhoA-JNK-C-Jun-MMP2 cascade by atorvastatin reduces osteosarcoma cell invasion. J Biol Chem 283: 30549-30556, 2008.

15. Fromigué O, Haÿ E, Modrowski D, Bouvet S, Jacquel A, Auberger P and Marie PJ: RhoA GTPase inactivation by statins induces osteosarcoma cell apoptosis by inhibiting $\mathrm{p} 42 / \mathrm{p} 44-\mathrm{MAPKs}-\mathrm{Bcl}-2$ signaling independently of BMP-2 and cell differentiation. Cell Death Differ 13: 1845-1856, 2006.

16. Yang GP and Lau LF: Cyr61, product of a growth factor-inducible immediate early gene, is associated with the extracellular matrix and the cell surface. Cell Growth Differ 2: 351-357, 1991.

17. Fromigue O, Lagneaux L and Body JJ: Bisphosphonates induce breast cancer cell death in vitro. J Bone Miner Res 15: 2211-2221, 2000.

18. Casey PJ and Seabra MC: Protein prenyltransferases. J Biol Chem 271: 5289-5292, 1996.

19. McTaggart SJ: Isoprenylated proteins. Cell Mol Life Sci 63: 255-267, 2006.

20. Hiraga T, Williams PJ, Mundy GR and Yoneda T: The bisphosphonate ibandronate promotes apoptosis in MDA-MB-231 human breast cancer cells in bone metastases. Cancer Res 61: 4418-4424, 2001.

21. Lee MV, Fong EM, Singer FR and Guenette RS: Bisphosphonate treatment inhibits the growth of prostate cancer cells. Cancer Res 61: 2602-2608, 2001.

22. Ory B, Heymann MF, Kamijo A, Gouin F, Heymann D and Redini F: Zoledronic acid suppresses lung metastases and prolongs overall survival of osteosarcoma-bearing mice. Cancer 104: 2522-2529, 2005.

23. Sonnemann J, Eckervogt V, Truckenbrod B, Boos J, Winkelmann W and van Valen F: The bisphosphonate pamidronate is a potent inhibitor of human osteosarcoma cell growth in vitro. Anticancer Drugs 12: 459-465, 2001.

24. Delarue FL, Adnane J, Joshi B, Blaskovich MA, Wang DA, Hawker J, Bizouarn F, Ohkanda J, Zhu K, Hamilton AD, et al: Farnesyltransferase and geranylgeranyltransferase I inhibitors upregulate RhoB expression by HDAC1 dissociation, HAT association and histone acetylation of the RhoB promoter. Oncogene 26: 633-640, 2007.

25. Sebti SM and Hamilton AD: Farnesyltransferase and geranylgeranyltransferase I inhibitors and cancer therapy: Lessons from mechanism and bench-to-bedside translational studies. Oncogene 19: 6584-6593, 2000.

26. Istvan E: Statin inhibition of HMG-CoA reductase: A 3-dimensional view. Atheroscler Suppl 4: 3-8, 2003.

27. Farwell WR, Scranton RE, Lawler EV, Lew RA, Brophy MT, Fiore LD and Gaziano JM: The association between statins and cancer incidence in a veterans population. J Natl Cancer Inst 100: 134-139, 2008. 
28. Beri A, Sural N and Mahajan SB: Non-atheroprotective effects of statins: A systematic review. Am J Cardiovasc Drugs 9: 361-370, 2009.

29. Gonyeau MJ and Yuen DW: A clinical review of statins and cancer: Helpful or harmful? Pharmacotherapy 30: 177-194, 2010

30. Babic AM, Kireeva ML, Kolesnikova TV and Lau LF: CYR61, a product of a growth factor-inducible immediate early gene, promotes angiogenesis and tumor growth. Proc Natl Acad Sci USA 95: 6355-6360, 1998.

31. Bleau AM, Planque N and Perbal B: $\mathrm{CCN}$ proteins and cancer: two to tango. Front Biosci 10: 998-1009, 2005

32. Menéndez JA, Mehmi I, Griggs DW and Lupu R: The angiogenic factor CYR61 in breast cancer: Molecular pathology and therapeutic perspectives. Endocr Relat Cancer 10: 141-152, 2003.

33. Jun JI and Lau LF: Taking aim at the extracellular matrix: $\mathrm{CCN}$ proteins as emerging therapeutic targets. Nat Rev Drug Discov 10: 945-963, 2011.

34. Hall-Glenn F and Lyons KM: Roles for CCN2 in normal physiological processes. Cell Mol Life Sci 68: 3209-3217, 2011

35. Chen CC and Lau LF: Functions and mechanisms of action of CCN matricellular proteins. Int J Biochem Cell Biol 41: 771-783, 2009.

36. Holbourn KP, Acharya KR and Perbal B: The CCN family of proteins: Structure-function relationships. Trends Biochem Sci 33: 461-473, 2008.

37. Leask A and Abraham DJ: All in the CCN family: Essential matricellular signaling modulators emerge from the bunker. J Cell Sci 119: 4803-4810, 2006.

38. Kubota S and Takigawa M: The role of CCN2 in cartilage and bone development. J Cell Commun Signal 5: 209-217, 2011.

39. Martirosyan A, Clendening JW, Goard CA and Penn LZ: Lovastatin induces apoptosis of ovarian cancer cells and synergizes with doxorubicin: Potential therapeutic relevance. BMC Cancer 10: 103, 2010.
40. Schmidmaier R, Baumann P, Bumeder I, Meinhardt G, Straka C and Emmerich B: First clinical experience with simvastatin to overcome drug resistance in refractory multiple myeloma. Eur J Haematol 79: 240-243, 2007.

41. van der Spek E, Bloem AC, Sinnige HA and Lokhorst HM: High dose simvastatin does not reverse resistance to vincristine, adriamycin and dexamethasone (VAD) in myeloma. Haematologica 92: e130-e131, 2007.

42. van der Spek E, Bloem AC, van de Donk NW, Bogers LH, van der Griend $\mathrm{R}$, Kramer MH, de Weerdt $\mathrm{O}$, Wittebol $\mathrm{S}$ and Lokhorst HM: Dose-finding study of high-dose simvastatin combined with standard chemotherapy in patients with relapsed or refractory myeloma or lymphoma. Haematologica 91: 542-545, 2006.

43. Marra M, Santini D, Meo G, Vincenzi B, Zappavigna S, Baldi A, Rosolowski M, Tonini G, Loeffler M, Lupu R, et al: Cyr61 downmodulation potentiates the anticancer effects of zoledronic acid in androgen-independent prostate cancer cells. Int J Cancer 125: 2004-2013, 2009.

44. Nguyen N, Kuliopulos A, Graham RA and Covic L: Tumor-derived Cyr61(CCN1) promotes stromal matrix metalloproteinase-1 production and protease-activated receptor 1-dependent migration of breast cancer cells. Cancer Res 66: 2658-2665, 2006.

45. Manara MC, Perbal B, Benini S, Strammiello R, Cerisano V, Perdichizzi S, Serra M, Astolfi A, Bertoni F, Alami J, et al: The expression of cen3 (nov) gene in musculoskeletal tumors. Am J Pathol 160: 849-859, 2002.

46. Perbal B, Zuntini M, Zambelli D, Serra M, Sciandra M, Cantiani L, Lucarelli E, Picci P and Scotlandi K: Prognostic value of CCN3 in osteosarcoma. Clin Cancer Res 14: 701-709, 2008. 